\title{
Daily Singing Practice as a Means of Improving Pulmonary Function and Quality of Life in Emphysema Patients
}

Anton Grasch, Theresa M. Boley, Joni Colle ${ }^{2}$, Joseph Q. Henkle ${ }^{3}$, Steve O. Todd ${ }^{4}$, Stephen O. Hazelrigg 4

1. 2. Division of Cardiothoracic Surgery, Southern Illinois University School of Medicine 3. Division of Pulmonary \& Critical Care, Southern Illinois University School of Medicine 4. Southern Illinois University School of Medicine

$\square$ Corresponding author: Anton Grasch, antongrasch@gmail.com Disclosures can be found in Additional Information at the end of the article

\section{Abstract}

Introduction: The purpose of this study was to determine if 10 minutes of singing every day might lead to improved pulmonary function and quality of life in chronic obstructive pulmonary disease (COPD) patients. Singing requires a controlled and precise use of respiratory musculature suggesting its daily use as a rehabilitation tool could help reduce respiratory symptoms.

Materials and Methods: This was a quasi-experimental pre-post test designed study lasting 12 weeks. The Springfield Committee for Research Involving Human Subjects approved the study, and all patients were provided written informed consent. Measurements were taken for actual and perceived respiratory function and quality of life. There were 25 patients who completed the study. All patients were enrolled in a rehabilitation program at a Phase 3 status. Patients were given an instructor-led demonstration as well as a pamphlet that guided them through a series of breathing and vocal warm-ups which, upon completion, they followed with singing a song. This process took approximately five minutes and was done morning and evening.

Results: Results for the study showed no mean changes beyond standard deviation in any of the measurements taken both in lung function and in quality of life. However, there was a statistically significant decrease in the Borg fatigue rating with a mean at baseline of $8.88+/-$ 3.76 and $7.60+/-2.61$ at follow-up $(\mathrm{p}=0.04)$.

Conclusions: The finding of less fatigue following 12 weeks of singing exercises suggests further research should be conducted to examine the therapeutic modality.

Received 03/01/2013

Review began 03/01/2013

Published 03/07/2013

(๑) Copyright 2013

Grasch et al. This is an open access article distributed under the terms of the Creative Commons Attribution License CC-BY 3.0., which permits unrestricted use, distribution, and reproduction in any medium, provided the original author and source are credited.
Categories: Internal Medicine

Keywords: copd, rehabilitation, singing, emphysema, spirometry

\section{Introduction}

Chronic obstructive pulmonary disease (COPD) is a debilitating disease of the lungs characterized by a narrowing of the airways on expiration. One of its most prevalent symptoms is dyspnea: the sensation of shortness of breath often preventing those affected by it from performing many normal daily activities. Due to its irreversible nature [1-2], COPD often has debilitating effects on the patient's everyday life. This can lead to depression, anxiety [3-4] 
and decreased quality of life [5-6] for COPD patients. The goal of therapy is to reduce associated disease symptoms [7]. The purpose of this study was to determine if 10 minutes of singing every day might lead to improved pulmonary function and quality of life in COPD patients.

Singing is an innate human ability. Using the body as its instrument, singing involves the passing of air from the lungs over the vocal cords in various semi-closed states to produce musical sounds. Proper sound production requires a controlled movement of this air from the lungs and the musical phrasing demands rapid and deep inhalations between the song's passages. To achieve this, the diaphragm and intercostals (both inspiratory and expiratory) are in a continuous cycling of sustained contraction [8]. This controlled and precise use of respiratory musculature suggests use of daily singing as a rehabilitation tool could improve lung function and efficiency. Daily vocal training could also lead to an improved quality of life for many patients as they experience the natural joy of singing [9-10]. Research in this area has thus far been limited with two previous studies providing only significant results in quality of life [11-12] as well as a slight (though not beyond standard deviation) increase to max expiratory pressure [11]. These previous studies requested that the patients sing twice a week and attend weekly group singing sessions. Through modifying this design to include daily singing, we aimed to cement these previous quality of life findings as well as see if more regular singing practice might achieve greater results.

\section{Materials And Methods}

\section{Study design}

This was a quasi-experimental pre/post-designed study with patients functioning as their own control. They were tested once at the beginning of the study and a second time 12 weeks later at the conclusion of the study.

\section{Inclusion/exclusion criteria}

The Springfield Committee for Research Involving Human Subjects approved the study, and thirty-one subjects consented to participate in the study. All patients had a diagnosis of COPD or emphysema without any other major concomitant respiratory illnesses and were enrolled in a pulmonary rehabilitation program at a Phase 3 status. Once patients consented to be included in the study, their diagnosis of COPD/emphysema was documented via their medical record. There was no age or gender criterion. The patient sample was pulled from patients at an affiliated hospital pulmonary rehabilitation program.

\section{Tools}

For the spirometry testing, we used a Sensormedic 20C Spirometer. The inspiratory and expiratory pressures were taken with a pressure manometer hooked up through a capped tpiece. Throughout all of the testing, a Sensormedic cardboard mouthpiece with bacteria filter was utilized for each patient. Quality of life was measured using the St. George's Respiratory Questionnaire (SGRQ) [13]. The SGRQ is a self-completed multiple-choice questionnaire involving the patients' perceived well-being in regards to comfort with their lung function during daily activities and how the disease affects their life. Three component scores are calculated: Symptoms, Activity, and Impacts from which a total score is derived. To measure dyspnea, we used the Borg scale [14]. The Borg scale is a numbered scale from 6 to 20 where an increase or decrease in the number will reveal an increase or decrease, respectively, in the severity of the patients' exertion or shortness of breath (for instance, 6: no exertion at all, 9: very light, 15: hard, etc). Borg testing occurred following the completion of the spirometry portion of the testing with the implication being the study participants rated their perceived fatigue following the energy, breathing or otherwise, that they exerted performing the 
spirometry. Brief Symptom Inventory 18 was also used [15]. This is an 18-item questionnaire, which measures depression, anxiety, and somatic symptoms, with a subscale score in each of those three categories. The General Severity Index is a composite score derived from this tool, which assesses the overall distress of the patient.

\section{Justification of the number of subjects}

In the Bonhila, et al. study [11], they found a significant change from pre-study to post-study on the quality of life assessment (SGRQ) with an effect size of 1.02. Given these results, it was calculated that we would have a statistical power in excess of 0.90 in this proposed pilot study with sample size $n=30$ to find a statistically significant difference, with alpha $=0.05$, on the SGRQ. This sample size yielded adequate statistical power $(>=0.80$ ) for effect sizes $>=0.54$. Additionally, the sample size $n=30$ allowed for reliable estimation of the effect sizes of the various objective pulmonary function measures which can be used in planning of the future studies. Due to withdrawal and exclusion, our sample size dropped to 25 patients; however, with this sample size, adequate power to find effect sizes of $>=0.59$ was still yielded.

\section{Clinical setting}

The testing for this study occurred at our school of medicine's pulmonary function laboratory. Follow-up meetings occurring throughout the study were conducted at two affiliated hospital rehabilitation facilities.

\section{Variables}

Data collected from the patients during the study included smoking status, level of adherence to daily singing schedule, routine exercise, any changes in medication, and any respiratory illness that may have occurred during the study.

\section{Procedures, interventions, schedule}

The patients were tested at the beginning of the study and then again 12 weeks later at the conclusion of study treatment. Both testing sessions included spirometry, measurement of inspiratory and expiratory pressures, and a quality of life assessment. A certified pulmonary function technologist and registered respiratory therapist with 25 years' experience trained the person performing the tests. The first round of testing included a one on one discussion with the patient, along with guiding them through the practice booklet they were asked to follow during their daily singing. Study participants were given the singing pamphlet that guided them through a series of breathing and vocal warm-ups, which upon completion, they were to follow with singing a song of their choice. Subjects were instructed to practice singing five minutes each morning and evening. They were given a calendar to mark sessions which they completed as they progressed through the study. Patients were contacted weekly in person or over the phone to answer questions and concerns regarding the singing pamphlet instructions and/or singing. Finally, patients were asked a series of post-study questions to assess their experience of the study.

\section{Planned data analysis}

Paired t-tests or Wilcoxon signed-rank tests, were used as appropriate, to examine the changes from pre- to post-intervention on the various outcome measures. Results were considered statistically significant for $\mathrm{p}<0.05$. All analyses were performed using SAS v9.3 software.

\section{Results}

\section{Study population}




\section{Cureus}

Thirty-one subjects diagnosed with emphysema consented to participate in the study, 25 of which completed the study, nine males/16 females. The mean age was $69.44+/-9.43$ (range: 47.00-84.00)

\section{Compliance}

Of the 168 possible singing sessions (twice a day over 12 weeks), the mean number of completed sessions was $146.95+/-13.97$ (range: $122-168$ ). Of the five minutes of time that was requested for each session to last, $84 \%$ of the participants sang five minutes or more with the remaining $16 \%$ singing less than five minutes.

\section{Lung function studies}

Forced expiratory volume l(FEV1), forced vital capacity (FVC), FEV1\% (FEV1/FVC), FEV1\% predicted, inspiratory pressure, and expiratory pressure measurements showed no significant changes from mean at baseline to follow-up.

\section{Measurement}

Forced expiratory volume 1 (FEV1)

Forced vital capacity (FVC)

Forced expiratory volume 1 \% (FEV1/FVC)

Forced expiratory volume 1\% predicted

Inspiratory pressure

Expiratory pressure

\section{Mean at Baseline}

$1.05+/-0.44$

$2.11+/-0.75$

$50.76+/-13.90$

$44.56+/-19.16$

$53.44+/-15.02$

$68.52+1-14.77$
Mean at Follow-up

t-value

1.59

0.12

$2.04+/-0.61$

1.28

$50.40+/-15.10$

0.32

0.75

$43.04+/-19.50$

$48.56+/-33.75$

$-.089$

0.38

$67.88+/-16.48$

0.81

\section{TABLE 1: Pulmonary Function Results}

\section{Quality of life studies and outgoing questioning}

The Borg Fatigue Rating did show a significant change at follow-up from the mean at baseline (7.60 vs 8.88 ). The SGRQ Total score and the Brief Symptom Inventory-18 Global Severity Index did not show any significant change at follow-up from baseline measurements. Additionally, there were no significant findings in the sub-scales of either of these questionnaires. Based on outgoing questioning, $84 \%$ of patients found the singing to be comforting or non-painful, $64 \%$ did not find the practice to be inconvenient, and 64\% were likely to continue it on their own. 


\section{Cureus}

\begin{tabular}{|c|c|c|c|c|}
\hline Measurements & Mean at Baseline & Mean at Follow-up & t-value & p-value \\
\hline Borg Fatigue & $8.88+/-3.76$ & $7.60+/-2.61$ & 2.2 & 0.04 \\
\hline BSI-18 & $52.56+/-9.11$ & $51.68+/-8.75$ & 0.67 & 0.51 \\
\hline SGRQ & $42.44+/-17.53$ & $39.87+/-13.86$ & 1.37 & 0.18 \\
\hline
\end{tabular}

\section{TABLE 2: Quality of Life Results}

BSI-18: Brief Symptom Inventory-18, SGRQ: St. George's Respiratory Questionnaire

\section{Discussion}

The guidelines set forth by the Global Initiative for Chronic Obstructive Pulmonary Disease state that the overall approach in the treatment of the patient with COPD "should be individualized to patient's symptoms and improvement of quality of life" [7]. The improvement in dyspnea achieved by current pulmonary rehabilitation yields positive results despite minimal changes to pulmonary function [1]. Previous studies [11-12] revealed a statistically significant increase in quality of life in COPD patients who sang twice weekly in a group setting. Our study utilizing daily singing, revealed a statistically significant improvement in the symptoms of COPD. While the question remains whether these results bear clinical significance, together these findings build support for the possibility of singing as a therapeutic tool in the treatment of COPD patients meriting further investigation.

Apparent in the guidelines to COPD treatment [7] is an implication that much, if not all, of the impaired or lost lung function in these patients is irreversible [1-2]. This inherent quality of the disease imposed a limitation to the possible improvement that could be recorded using spirometry and lung function testing both in our study and previous studies. Also, further limiting the study is a predisposition in the COPD patient population to have continued lung function deterioration over time. This could easily have disrupted the data set, reducing spirometry results by means outside of the causality of the therapeutic intervention being tested. Though literature has addressed expected changes in the lung function of COPD patients over one year, no study has looked at the disease course over shorter time intervals, such as the three months our study lasted. The small size of the local COPD population prevented incorporation of a control group into this study; however, expanded future studies could correct for this first by utilizing a control group with no intervention at all apart from the phone contact or with an intervention, such as relaxation in order to track lung function deterioration over the study's duration in the general COPD population as compared to the tested/singing group. The study time interval could also be lengthened. Our study was conducted from late spring (May) through the summer (August); extending the duration of the study could help determine whether or not deterioration in lung function is due to a transient drop secondary to acute illness or seasonal effects or due to permanent and chronic loss of function.

\section{Singing practice in emphysema patients}

Other major limitations to this study were the sample size, the locale of the study and exclusion criteria. In order to maintain the power of the study, the small sample size caused the need to retain patients who were both non-compliant and became ill during the study. This possibly resulted in a skewing of the data set towards loss of lung function in the patients' status since non-compliance to treatment, and especially illness, can result in deterioration. In future 
studies, sample size could be greatly expanded by not only increasing the number of locations but also by altering or removing certain exclusionary criteria. Removing exclusions on concomitant illness and lung function status would help to expand the sample while also helping to possibly reveal the benefit of singing in the true COPD population, which includes a spectrum of lung function and accompanying concomitant illness.

Current treatment geared towards improving COPD patients' symptoms and quality of life is focused heavily on pulmonary rehab, often using physical exercise programs, including walking, biking, and stability balls [1, 7]. Singing could be a useful tool to add to this repertoire in that it is highly portable, allowing the patient to perform elements of rehabilitation at home or when traveling. This helps provide greater independence to the patient which is a common and important therapeutic goal of most rehabilitation. Singing would also be a rehabilitation tool that could be utilized by any patient, regardless of mobility status. Finally, as revealed by outgoing questioning, singing is convenient for a majority of patients and is enjoyable enough to the patient that a majority of our study participants were planning to continue singing on their own.

\section{Conclusions}

Patients with severe COPD who participated in regular singing 10 minutes daily over 12 weeks demonstrated a significant decrease in their Borg fatigue rating, suggesting an improvement in the patients' perception of their dyspnea. While there was no significant change in forced vital capacity (FVC), forced expiratory volume 1 (FEV1), inspiratory or expiratory pressures, this study caused little inconvenience or discomfort to the patient and improved the symptoms that global guidelines set forth as the goal for COPD treatment. This suggests further research should be conducted to examine singing as a rehabilitation modality for the treatment of emphysema.

\section{Additional Information \\ Disclosures}

Human subjects: Consent was obtained by all participants in this study. The Springfield Committee for Research Involving Human Subjects issued approval \# 12-535 (electronic protocol \#); \# 09-017 (original protocol \# upon article submission). Animal subjects: All authors have confirmed that this study did not involve animal subjects or tissue. Conflicts of interest: In compliance with the ICMJE uniform disclosure form, all authors declare the following: Payment/services info: All authors have declared that no financial support was received from any organization for the submitted work. Financial relationships: All authors have declared that they have no financial relationships at present or within the previous three years with any organizations that might have an interest in the submitted work. Other relationships: All authors have declared that there are no other relationships or activities that could appear to have influenced the submitted work.

\section{References}

1. Celli BR, MacNee W, Agusti A, Anzueto A, Berg B, Buist AS, Calverley PMA, Chavannes N, Dillard T, Fahy B, Fein A, Heffner J, Lareau S, Meek P, Martinez F, McNicholas W, Muris J, Austegard E, Pauwels R, Rennard S, Rossi A, Siafakas N, Tiep B, Vestbo J, Wouters E, ZuWallack R: Standards for the diagnosis and treatment of patients with COPD: a summary of the ATS/ERS position paper. Eur Respir J . 2004, 23:932-946.

2. Nici L, Donner C, Wouters E, Zuwallack R, Ambrosino N, Bourbeau J, Carone M, Celli B, Engelen M, Fahy B, Garvey C, Goldstein R, Gosselink R, Lareau S, Maclntyre N, Maltais F, Morgan M, O'Donnell D, Prefault C, Reardon J, Rochester C, Schols A, Singh S, Troosters T: 
American Thoracic Society/European Respiratory Society Statement on Pulmonary Rehabilitation. Am J Respir Crit Care Med . 2006, 173:1390-1413.

3. Dowson CA, Cuijer RG, Mulder RT: Anxiety and self management behavior in chronic pulmonary disease: what has been learned?. Chron Respir Dis. 2004, 1:213-220.

4. Singer HK, Ruchinskas RA, Riley KC: The psychological impact of end-stage lung disease . Chest. 2001, 120:1246-1252.

5. Cully JA, Graham DP, Stanley MA, Ferguson CJ, Sharafkhaneh A, Souchek J, Kunik ME: Quality of life in patients with chronic pulmonary disease and comorbid anxiety or depression. Psychosomatics. 2006, 47:312-9.

6. Ferrer M, Alonso J, Morera J, Marrades R, Khalaf A, Aguar C, Plaza V, Prieto L, Anto J: Chronic obstructive pulmonary disease stage and health related quality of life. Ann Int Med . 1997, 127:1072-1079.

7. Pauwels RA, Buist AS, Calverley PM, Jenkins CR, Hurd SS, the GOLD Scientific Committee: Global strategy for the diagnosis, management, and prevention of chronic obstructive pulmonary disease. NHLBI/WHO Global Initiative for Chronic Obstructive Lung Disease (GOLD) Workshop summary. Am J Respir Crit Care Med . 2001, 163:1256-1276.

8. Sundberg J: Breathing Behavior During Singing. The NATS Journal. 1993, 49:2-9, 49-51.

9. Kenny DT, Faunce G: The impact of group singing on mood, coping and perceived pain in chronic pain patients attending a multidisciplinary pain clinic. J Music Ther. 2004, 41:241-58.

10. Grape C, Sandgren M, Hansson LO, Ericson M, Theorell T: Does singing promote well being ? An empirical study of professional and amateur singers during a singing lesson. Integr Physiol Behav Sci. 2003, 38:65-74.

11. Bonilha AG, Onofre F, Vieira ML, Prado MY, Martinez JA: Effects of singing classes on pulmonary function and quality of life of COPD patients. Int J Chron Obstruct Pulmon Dis. 2009, 4:1-8.

12. Lord VM, Cave P, Hume VJ, Flude EJ, Evans A, Kelly J, Po!key M, Hopkinson NS: Singing teaching as a therapy for chronic respiratory disease - a randomised controlled trial and qualitative evaluation. BMC Pulm Med. 2010, 10:41.

13. Jones PW, Quirk FH, Baveystock CM, Littlejohns P: A self-complete measure for chronic airflow limitation - the St George's Respiratory Questionnaire. Am Rev Respir Dis . 1992, 145:1321-1327.

14. Borg GA: Psychophysical bases of perceived exertion . Med Sci Sports Exerc. 1982, 14:377-381.

15. Derogatis, LR: Brief Symptom Inventory 18 (BSI-18): Administration, Scoring, and Procedures Manual. NCS Pearson, Inc, 2001. 